\title{
Angiogenic Peptide Nanofibers Improve Wound Healing in STZ- Induced Diabetic Rats
}

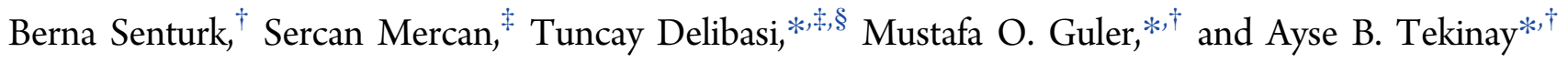 \\ ${ }^{\dagger}$ Institute of Materials Science and Nanotechnology, National Nanotechnology Research Center (UNAM), Bilkent University, Ankara \\ 06800, Turkey \\ ${ }^{\ddagger}$ Department of Endocrinology and Metabolism, ADACELL Cell Therapy, Regenerative Medicine and Research Hospital Etlik \\ Polyclinic, Ankara, 06010, Turkey \\ ${ }^{\S}$ Department of Internal Medicine, School of Medicine (Kastamonu), Hacettepe University, Ankara, 06100, Turkey
}

Supporting Information

ABSTRACT: Low expressions of angiogenic growth factors delay the healing of diabetic wounds by interfering with the process of blood vessel formation. Heparin mimetic peptide nanofibers can bind to and enhance production and activity of major angiogenic growth factors, including VEGF. In this study, we showed that heparin mimetic peptide nanofibers can serve as angiogenic scaffolds that allow slow release of growth

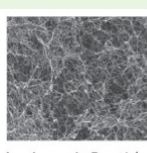
Angiogenic Peptide Nanofiber

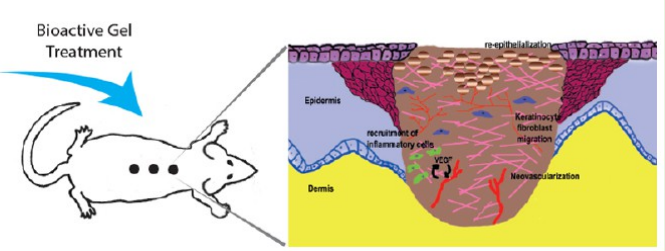
factors and protect them from degradation, providing a new therapeutic way to accelerate healing of diabetic wounds. We treated wounds in STZ-induced diabetic rats with heparin mimetic peptide nanofibers and studied repair of full-thickness diabetic skin wounds. Wound recovery was quantified by analyses of reepithelialization, granulation tissue formation and blood vessel density, as well as VEGF and inflammatory response measurements. Wound closure and granulation tissue formation were found to be significantly accelerated in heparin mimetic gel treated groups. In addition, blood vessel counts and the expressions of alpha smooth muscle actin and VEGF were significantly higher in bioactive gel treated animals. These results strongly suggest that angiogenic heparin mimetic nanofiber therapy can be used to support the impaired healing process in diabetic wounds.

KEYWORDS: heparin mimetic, diabetic wound healing, peptide amphiphiles, angiogenesis, VEGF expression

\section{INTRODUCTION}

Diabetes mellitus is a chronic disease caused either by the destruction of insulin-producing beta cells in the pancreas (type-1 diabetes) or insufficient response to secreted insulin (type-2 diabetes). ${ }^{1}$ The prevalence of both of types of diabetes has been on the rise, and the disease may soon become one of the foremost public health problems that the world will face in the decades ahead. ${ }^{2}$ In addition to the direct consequences of a malfunctioning carbohydrate metabolism, diabetes is associated with various secondary complications, including severe chronic ulcers that occur on the feet and sometimes necessitate limb amputation. ${ }^{3}$ The healing of other injuries is also delayed in diabetic patients, and the increase in the prevalence of diabetes has made it an important necessity to develop appropriate wound dressings for diabetic wounds. Clinical and experimental studies showed that the four classical phases of wound healing do not occur in their typical order and fail to fully perform their function in diabetic wounds. ${ }^{4}$ In chronic wounds, the impairment of the healing process is mostly related to degradation or diminished expression of growth factors, which hinders the generation of blood vessels and consequently prevents the transport of oxygen and nutrients to the wound area. ${ }^{5,6}$ A variety of angiogenic growth factors are involved in the wound healing process, the most important of which is vascular endothelial growth factor (VEGF); however, the expression of VEGF is dramatically reduced in diabetic wounds. ${ }^{7,8}$ VEGF is vital in stimulating cell proliferation and strongly influence endothelial tube formation. It has been demonstrated that the treatment of wound site with VEGF accelerates wound healing via improved granulation tissue formation in the rabbit ear and induce angiogenesis in diabetic mice. $^{9,10}$

Heparan sulfate proteoglycans (HSPGs) are members of glycosaminoglycan (GAG) family which are found in the extracellular matrix and plasma membrane. ${ }^{11}$ HSPGs modulates its functions by interacting with a broad variety of angiogenic growth factors. ${ }^{12}$ The heparin binding affinity is critical for stimulation of vasculogenesis and angiogenesis and VEGF plays an especially vital role in this context. Although HSPGs can be administered directly to the wound site to improve wound healing, they have limitations such as vulnerability to enzymatic degradation, batch-to-batch variation and high heterogeneity. ${ }^{13}$ It has also been proposed that the failure of treatments of diabetic wounds using exogenous growth factors such as VEGF

Received: May 2, 2016

Accepted: June 6, 2016

Published: June 16, 2016 
in clinical trials, might be due to instability of natural materials in enzyme rich environment. ${ }^{14}$ However, recent advances in material sciences and polymer chemistry have resulted in the development of synthetic biomimetic peptide fibers, an entire class of new nanomaterials that can mimic the bioactivity of natural materials. Peptide amphiphiles (PAs) are self-assembling biodegradable materials that have a potential to replicate the function of native extracellular matrix, and can therefore be used to enhance the angiogenesis process in diabetic wounds with greater control over growth factor release kinetics. ${ }^{15}$

Previously, we reported heparin mimetic PAs (GAG-PA/K$\mathrm{PA})$ that are able to induce angiogenesis without the addition of heparin or exogenous growth factors under both in vitro and in vivo conditions. ${ }^{16}$ Furthermore, it has been shown that GAG$\mathrm{PA} / \mathrm{K}-\mathrm{PA}$ bioactive nanofibers display strong binding affinities to VEGF, hepatocyte growth factor (HGF), and fibroblast growth factor-2 (FGF-2). The high binding capacity of nanofibers is particularly valuable since they protect growth factors from degradation and provide a suitable cellular microenvironment for signaling. Because angiogenesis is the key component of the wound healing process and is regulated by of HSPGs, heparin mimetic PAs may also enhance blood vessel formation in diabetic patients.

Here, we show the effect of heparin mimetic PA scaffolds on the recovery of diabetic wounds. We analyzed reepithelialization, granulation tissue formation, blood vessel density, and inflammatory response of full skin thickness wounds inflicted on STZ-induced diabetic rats. In addition, we investigated impact of heparin mimetic gel treatment on VEGF expression.

\section{MATERIALS AND METHODS}

2.1. Materials. 9-fluorenylmethoxycarbonyl (Fmoc) and tertbutoxycarbonyl (Boc) protected amino acids, [4-[a-(20,40-dimethoxyphenyl) Fmocaminomethyl]enoxy]acetamidonorleucyl-MBHA resin (Rink amide MBHA resin), Fmoc-Asp $(\mathrm{OtBu})-\mathrm{Wang}$ resin and 2-(1HBenzotriazol-1-yl)-1,1,3,3-tetramethyluronium hexafluorophosphate (HBTU) were purchased from NovaBiochem (8.51006.0100) and ABCR (AB172551). Antibodies were purchased from Abcam and Millipore. Streptozocin (STZ) were purchased from Sigma-Aldrich (18883-66-4) and biopsy punch from Microtek Medical (Zutphen, NL). Animals were anaesthetized with $2 \%$ Alfazyne (xylazine hydrochloride) and 10\% Alfamine (ketamine hydrochloride). All other chemicals and materials used in this study were analytical grade and obtained from Invitrogen, Fisher, Merck, Alfa Aesar, and SigmaAldrich.

2.2. Peptide Synthesis and PA Gel Formation. Peptide amphiphile (PA) molecules used in this study were synthesized by solid phase peptide synthesis, as previously described. ${ }^{16}$ Briefly, rink amide MBHA resin (for GAG-PA, K-PA) and Fmoc-Glu(OtBu)-Wang resin (for E-PA) were used as solid support. Amino acid couplings were performed with two equivalents of Fmoc protected amino acid, 1.95 equiv HBTU and 3 equiv of N,N-diisopropylethylamine (DIEA) for $2 \mathrm{~h}$. Fmoc removal was performed with a $20 \%$ piperidine/ dimethylformamide solution (DMF) for $20 \mathrm{~min} .10 \%$ acetic anhydride solution in DMF was used to block remaining free amine groups after amino acid coupling. The $p$-sulfobenzoic acid was added to the side chain of lysine to synthesize sulfonated PAs. During the synthesis of GAG-PA, Mtt removal was performed by shaking resins with TFA:TIS: $\mathrm{H}_{2} \mathrm{O}: \mathrm{DCM}$ in the ratio of 5:2.5:2.5:90. PAs were cleaved from the resin with a mixture of TFA:TIS: $\mathrm{H}_{2} \mathrm{O}$ in a ratio of 95:2.5:2.5 for $2 \mathrm{~h}$. PAs were characterized by liquid chromatography-mass spectroscopy (LC-MS). A gradient of water ( $0.1 \%$ formic acid or $\left.0.1 \% \mathrm{NH}_{4} \mathrm{OH}\right)$ and acetonitrile $\left(0.1 \%\right.$ formic acid or $\left.0.1 \% \mathrm{NH}_{4} \mathrm{OH}\right)$ was used as the mobile phase. To purify the peptides, Agilent preparative reverse-phase HPLC system equipped with Zorbax
Extend-C18 $21.2 \times 150 \mathrm{~mm}$ column and Zorbax SB-C8 $21.2 \times 150$ $\mathrm{mm}$ column were used for basic and acidic conditions, respectively.

GAG-PA, E-PA, and K-PA were individually dissolved in distilled water and sonicated for $10 \mathrm{~min}$ prior to use. Bioactive gel formation was performed through the mixing of GAG-PA and K-PA at a 1:2 molar ratio, while nonbioactive gels were formed by mixing E-PA and K-PA at a 1:1 molar ratio. Peptide solutions were adjusted to $\mathrm{pH} 7.4$ prior to use. Net charges of the system had negative potentials for both bioactive and nonbioactive nanofibers. Peptide mixtures were prepared sterilized under UV light just before the experiments.

2.3. Characterization of Peptide Amphiphile Molecules. Scanning electron microscopy (SEM) samples were prepared by incubating, $1 \mathrm{wt} \%$ of GAG-PA/K-PA or E-PA/K-PA gels for $20 \mathrm{~min}$ on silicon wafer. Samples were then dehydrated by immersion into increasing concentrations of ethanol, dried in a critical point drier (Tousimis, Autosamdri-815B), and coated with a $6 \mathrm{~nm} \mathrm{Au-Pd} \mathrm{layer}$ prior to analysis.

Circular dichroism (CD) experiments were performed using a J-815 Jasco spectrophotometer. $3.6 \times 10^{-4} \mathrm{M}$ aqueous solutions were diluted from $1 \mathrm{mM}$ stock peptide solutions for $\mathrm{CD}$ spectroscopy. Measurements were acquired at room temperature with $500 \mathrm{~nm} / \mathrm{min}$ scanning speed, $1 \mathrm{~nm}$ bandwidth and $0.1 \mathrm{~nm}$ data pitch within the data interval of 300 to $190 \mathrm{~nm}$. Three consecutive measurements were averaged for each sample. The results were converted to and represented as molar ellipticity.

Oscillatory rheology measurements were performed with an Anton Paar Physica RM301 rheometer operating with a $25 \mathrm{~mm}$ parallel plate configuration at $21^{\circ} \mathrm{C}$. One wt \% of GAG-PA/K-PA or E-PA/K-PA with total volume of $300 \mu \mathrm{L}$ was loaded on the center of the lower plate. The upper plate was brought to $0.5 \mathrm{~mm}$ gap position and the gel was incubated for $10 \mathrm{~min}$ before measurement. Storage moduli $\left(G^{\prime}\right)$ and loss moduli $\left(G^{\prime \prime}\right)$ values were scanned at an angular frequency of 100 to $0.1 \mathrm{rad} / \mathrm{s}$, with $0.5 \%$ shear strain. Three repeats were performed for each gel.

2.4. Animal Models. Male Sprague-Dawley (SD) rats (weight, 300 to $350 \mathrm{~g}$; age, 12 to 14 weeks) were obtained from ADACELL Laboratory (Ankara, Turkey). Rats were exposed to a $12 \mathrm{~h}$ light-dark cycle and fed a standard laboratory diet with food and water provided ad libitum. The local Animal Experiments Committee approved all procedures and study design. Animals were fasted overnight and given an intraperitoneal injection of STZ (Sigma-Aldrich, St. Louis, MO) at a dose of $45 \mathrm{mg} / \mathrm{kg}$ body weight in $0.1 \mathrm{~mol} / \mathrm{L}$ sodium citrate buffer, $\mathrm{pH} 4.5$ for the induction of diabetes. Blood glucose concentrations were monitored weekly by a GlucoDr Super Sensor Blood Glucose Test Meter (Korea) from tail vein blood. Animals that exhibited blood glucose levels over $20 \mathrm{mmol} / \mathrm{L}$ throughout the induction period were considered to be stably diabetic and used for wound healing experiments. Approximately $95 \%$ of animals were become stably hyperglycaemic after STZ injection.

Three weeks after STZ injection, 60 male Sprague-Dawley (SD) rats were randomly divided into 3 groups for GAG-PA/K-PA treatment $(n=5$ each for D0, D7, D9, and D14), PBS control treatment ( $n=5$ each for D0, D7, D9 and D14) and E-PA/K-PA treatment ( $n=5$ each for D0, D7, D9, and D14) (Figure 2D). Rats were anesthetized by intraperitoneal injection of xylazine hydrochloride (Alfazyne, $10 \mathrm{mg} / \mathrm{kg}$ ) and ketamine hydrochloride (Alfamine, $25 \mathrm{mg} / \mathrm{kg}$ ). Prior to creation of wounds, dorsal hairs of diabetic mice were completely shaved and the underlying skin was sterilized with povidone iodide. Three full-thickness wounds of $6 \mathrm{~mm}$ diameters were created on the dorsal skin using a biopsy skin punch for all treatment groups (Microtek Medical, Zutphen NL). Bioactive and nonbioactive gels were prepared immediately prior to their topical application. $1 \%$ $(\mathrm{w} / \mathrm{v})$ of gels or saline for $300 \mu \mathrm{L}$ were applied directly onto each wound area and covered with Octacare nonwoven wound dressing (OCTAMED). This wound dressing was preferred due to its elastic, air permeable, sterile, nonallergenic, long-term powerful adhesion, and absorbent band properties that can be easily removed from wound. The optimization studies showed that reapplication of gel on day 2 accelerated wound healing in diabetic rats. Therefore, gels (and PBS) were reapplied and the wounds were redressed 2 days postwounding. 
A
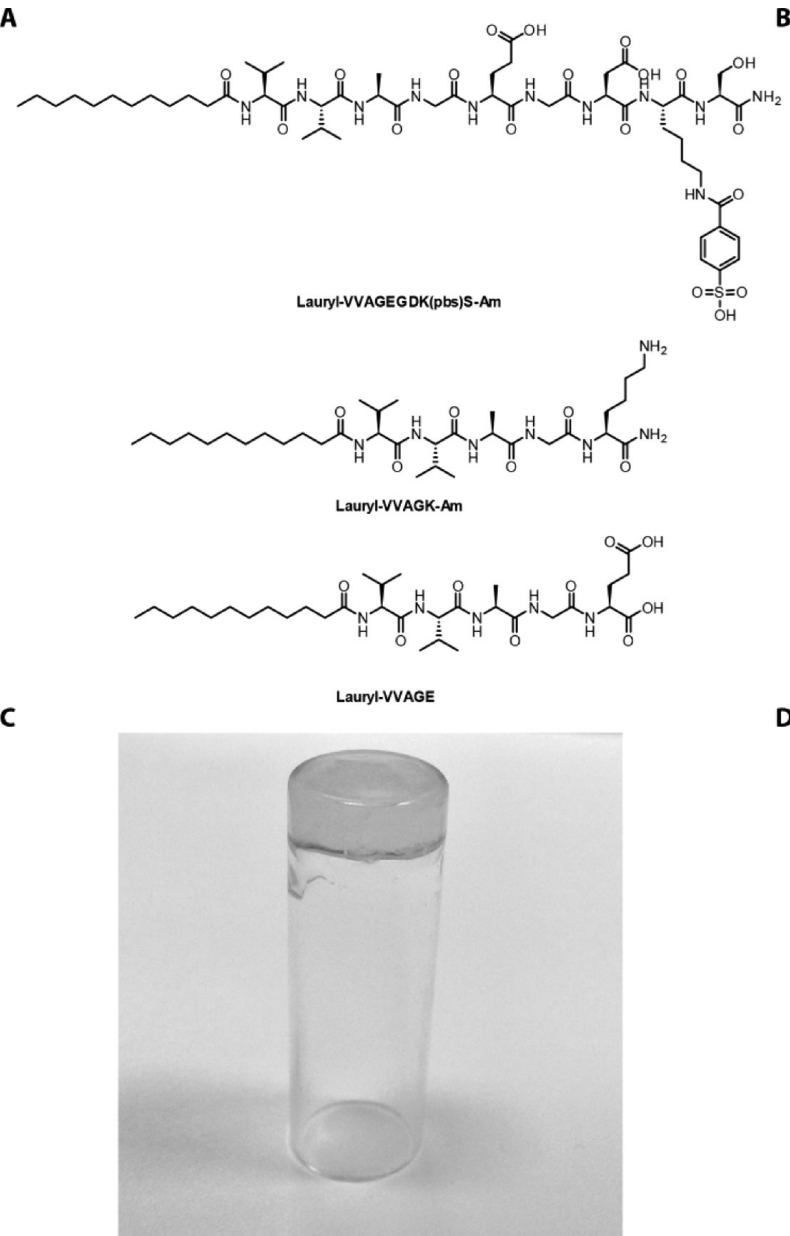

B

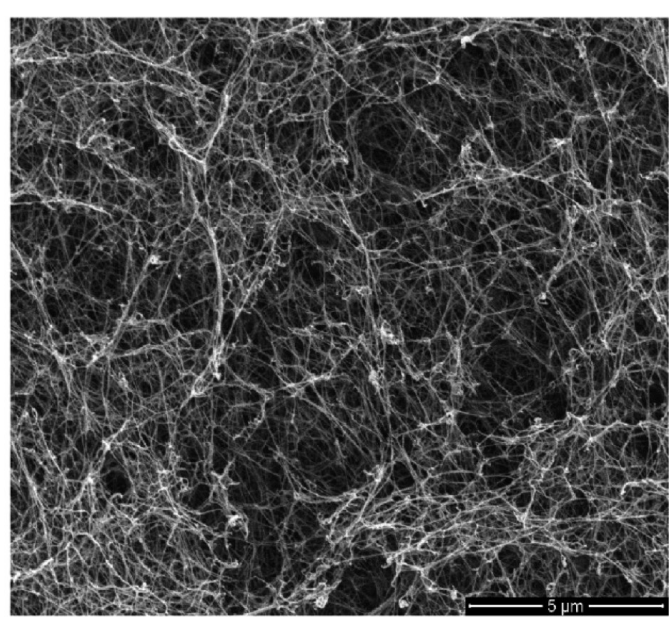

D

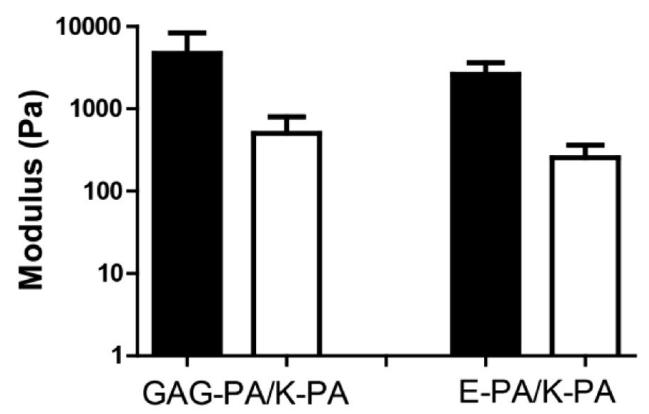

Figure 1. (A) Self-assembled peptide amphiphile (PA) nanofibers. Chemical structures of GAG-PA, K-PA, and E-PA are shown. (B) SEM image of GAG-PA/K-PA gel reveals the ECM like morphology of angiogenic scaffolds. (C) Photograph of the angiogenic gel formed by GAG-PA and K-PA peptide solutions. (D) Storage and loss moduli of PA gels were measured at $0.5 \%$ of shear strain and scanned from 100 to $0.1 \mathrm{rad} / \mathrm{s}$ of angular frequency. Rheology results showed gelation as a result of nanofibrous network formation by GAG-PA/K-PA and E-PA/K-PA at pH 7.4.

Thereafter, the animals were individually caged. At each experimental end point (day $0,3,7,9$, and 14), animals were sacrificed and their tissues were extracted for subsequent analyses. Body weights were measured using an electronic balance on the day of wounding (day 0 ), as well as on days $3,7,9,12$, and 14 post wounding.

2.5. Measurement of Wound Area. Wound margins were traced on days $3,7,9$, and 14 post wounding by using transparent papers. The wound tracing was performed only euthanized rats. The boundary of wound lines was traced from edge of the healthy epithelium. Wound area was traced on the graph paper and calculated with ImageJ.

2.6. Histological Analysis. On days 3, 7, 9, and 14, diabetic rats were sacrificed and their skin tissues were fixed with $10 \%$ formalin and embedded in paraffin. Tissue sections $(5 \mu \mathrm{m})$ were deparaffinized in xylene, rehydrated through graded alcohols to distilled water, and stained with hematoxylin and eosin (H\&E). Collagen formation was evaluated by Masson's trichrome staining. For this purpose, sections were initially deparaffinized, rehydrated, placed in Weigert's hematoxylin, and rinsed under tap water for $10 \mathrm{~min}$. Sections were then immersed into phosphomolybdic acid, aniline blue, acetic acid and ethanol in the order provided.

All quantifications associated with $\mathrm{H} \& \mathrm{E}$ staining were performed using ImageJ. Images of H\&E stained tissue sections were taken with $50 \times$ magnifications by an up-right microscope (Zeiss Axio Scope A1) and visually inspected for epithelialization scoring ( 0 absent, 1 weak, 2 moderate, 3 complete re-epithelialization of the wound).

Scoring was performed by three researchers who were blind to the study design. The thickest distance between the top and the bottom of the epithelium was measured for the quantification of epithelial thickness. Additionally, the distance between epithelial tips were calculated from images via measurement of length of gap between ends of epithelial tissue. The epithelial distance measurement was performed at days 3, 7, and 9 with Image J tool. On day 14, reepithelialization was complete for most of the samples; as such, distance was not calculated for this day. The whole granulation tissue area was traced and measured with ImageJ. Monocyte/macrophage staining images were taken with $100 \times$ magnification by up-right microscope.

2.7. Immunohistochemical Staining. Tissue sections were deparaffinized and hydrated in graded ethanol solutions and distilled water. Endogenous peroxidase was inactivated with $3 \%$ hydrogen peroxide for $10 \mathrm{~min}$ at room temperature. Nonspecific binding sites were blocked with $10 \%$ normal goat serum for $2 \mathrm{~h}$ at room temperature. Antigens were then detected by indirect binding with primary antibodies and HRP-conjugated secondary antibodies. Serial sections were immunolabeled with anti-von Willebrand factor antibody (1:400 dilution; ab6994, Abcam), alpha smooth actin antibody (1:500 dilution; ab76549, Abcam) or CD68 antibody (1:200 dilution; ab31630, Abcam) to evaluate the degree of healing in wound tissues. Sections were developed in 3,3'-diaminobenzidine (DAB) and counterstained with hematoxylin.

The number of blood vessels was counted from at least 6 randomly selected fields in each section; sections were immunolabeled with antivon Willebrand Factor (vWF) antibody and quantified by two independent observers under $200 \times$ magnification. Quantifications were performed with positive staining of vWF staining + lumen 

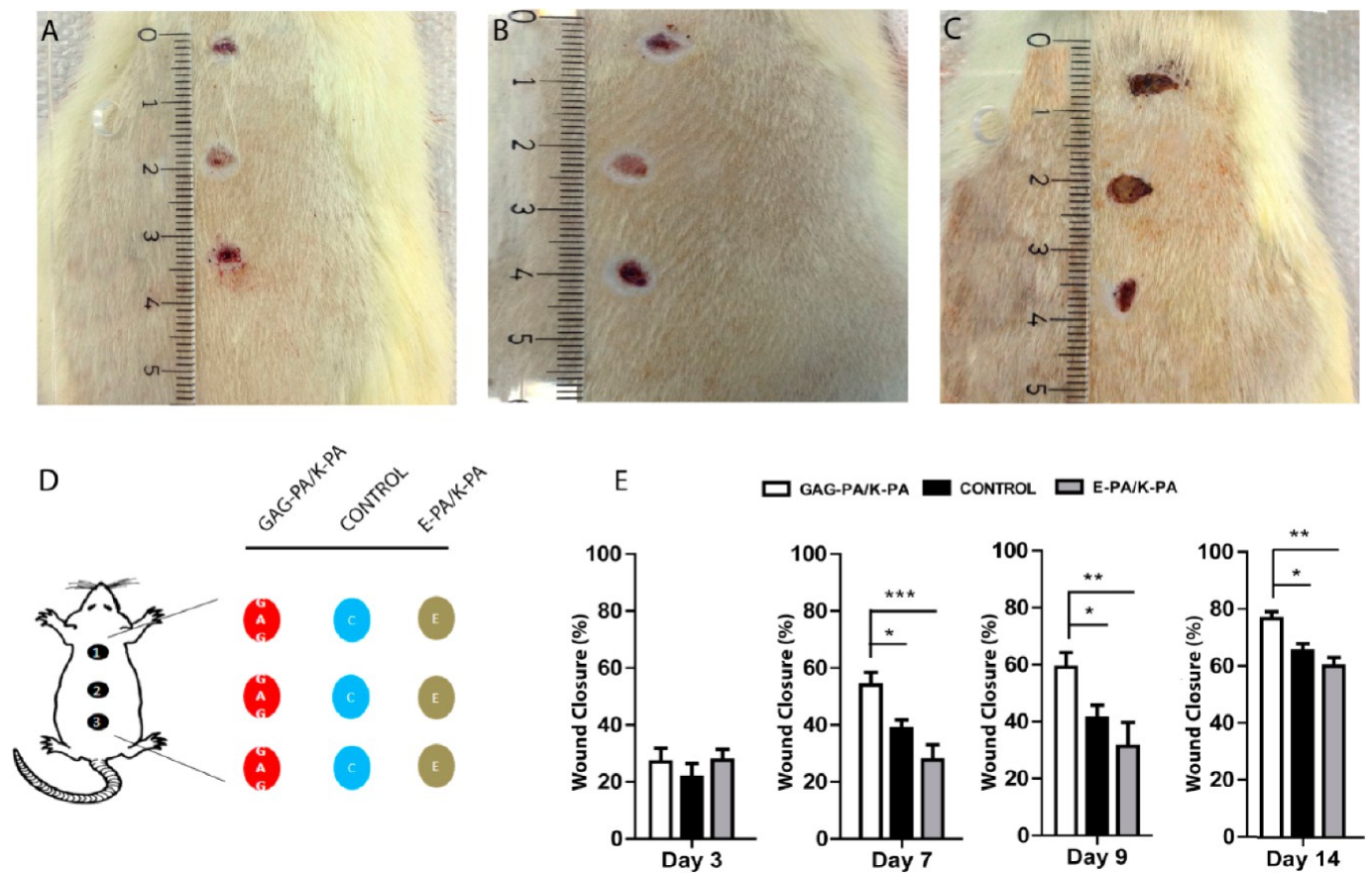

Figure 2. Wound closure was accelerated in GAG-PA/K-PA-treated wounds compared to controls. Photos of wounds (day 9) treated with (A) GAG-PA/K-PA, (B) PBS-control, and (C) E-PA/K-PA. (D) Wound locations (1, 2, and 3) and treatments where C indicates PBS control; E, nonbioactive gel; and GAG, glycosaminoglycan mimetic gel; each animal received a single treatment type across all three wounds. (E) Percentage wound closure areas at days 3, 7, 9, and 14. One-way ANOVA analysis was performed. Statistical differences are indicated as $* p<0.05$, ** $p<0.01$, and $* * * p<0.001, n=15$.

formation. ImageJ analysis system was used to calculate the number of vessels in each field.

2.8. Western Blot Analysis. Tissue samples were collected from the same animals used in histological analysis at day 7 and $14 ; n=3$ animals were used for each GAG-PA/K-PA, E-PA/K-PA and PBS treatment. Granulation tissues were homogenized in lysis buffer (octyl $\alpha$-D-glucopyranoside protease; ab144799, Abcam, and Protease Inhibitor Cocktail, Thermo Scientific). Protein concentrations were determined using a BCA Protein Assay Kit. Equal amounts of proteins $(50 \mu \mathrm{g}, 15 \mu \mathrm{L})$ were separated by NuPAGE Tris-Acetate gels under denaturing and nonreducing conditions and then transferred to a PVDF membrane. The membrane was blocked with $5 \%$ nonfat milk in TBST at room temperature for $1 \mathrm{~h}$ and then incubated with antiVEGF antibody (Abcam, ab1316, 1:400) at $4{ }^{\circ} \mathrm{C}$ overnight. After washing in TBST, the blots were incubated with a HRP-coupled secondary antibody (Millipore, 12-349 Goat Anti Mouse IgG, $1: 1000)$. Signals were visualized using a chemiluminescent signal enhancement system (Invitrogen, Novex ECL). GAPDH was used as the internal control and treated with the same protocol (Millipore, MAB374, 1:500). Gels were visualized by a Bio Rad VersaDoc MP4000 imaging system and protein concentrations in gel slabs were quantified using ImageJ. Intensities of VEGF bands were normalized by GAPDH.

2.9. Statistical Analysis. Statistical analyses were performed using GraphPad Prism 5. One-way and two-way ANOVA was used to compare the differences between the groups and Dunn's multiple comparison test was used for posthoc correction. Error bars indicate the standard error of the mean.

\section{RESULTS}

3.1. Synthesis and Characterization of PAs. Peptide amphiphiles (PA) used in the formation of bioactive GAG-PA/ K-PA and nonbioactive E-PA/K-PA gels were synthesized by solid phase peptide synthesis method. Lauryl-VVAGEGDK(pbs)S-Am (GAG-PA) was previously designed to mimic sulfated GAGs by presenting sulfonate, hydroxyl and carboxylate groups, while Lauryl-VVAGE-Am (E-PA) was used as the nonbioactive control peptide (Figure 1A). Positively charged Lauryl-VVAGK-Am (K-PA) was used to induce nanofiber formation through electrostatic interaction with negatively charged GAG-PA or E-PA. Peptide amphiphiles were characterized by LC-MS and purified with preparative HPLC (Figure S1). TEM images of these mixtures demonstrated that nanofiber structures were formed following the mixing of two oppositely charged peptides amphiphiles, with average diameters of $10-20 \mathrm{~nm}$, and lengths up to several micrometers. ${ }^{16}$ SEM images revealed that the peptide nanofibers formed by the PA molecules exhibited a nanofibrous network that showed similar morphology to natural $\mathrm{ECM}^{17}$ (Figure S2, Figure 1B). To analyze secondary structure of nanofiber formation of peptide amphiphiles and their mixtures, circular dichroism (CD) spectroscopy was employed. ${ }^{18} \mathrm{CD}$ results showed that the nanofibrous structures formed by the PA molecules contained $\beta$-sheet structures as evidenced by absorbance maximum at around $200 \mathrm{~nm}$ and minimum at around $220 \mathrm{~nm}$ (Figure S3). Oscillatory rheology was performed to investigate the mechanical properties of peptide amphiphile gels (Figure 1D). The concentrations of the gels were identical to these used in in vivo experiments. Storage $\left(G^{\prime}\right)$ and loss $\left(G^{\prime \prime}\right)$ moduli were scanned from 100 to $0.1 \mathrm{rad} / \mathrm{s}$ of angular frequency and with $0.5 \%$ shear strain. Rheology results indicated that both bioactive and nonbioactive PA molecules formed gels as storage moduli were higher than loss moduli. The visual observation of PA mixtures also supported the gel formation (Figure 1C). Additionally, measurements showed that GAG-PA/K-PA and E-PA/K-PA gels had similar mechanical properties with stiffness values within a range of 3-8 kPa. 

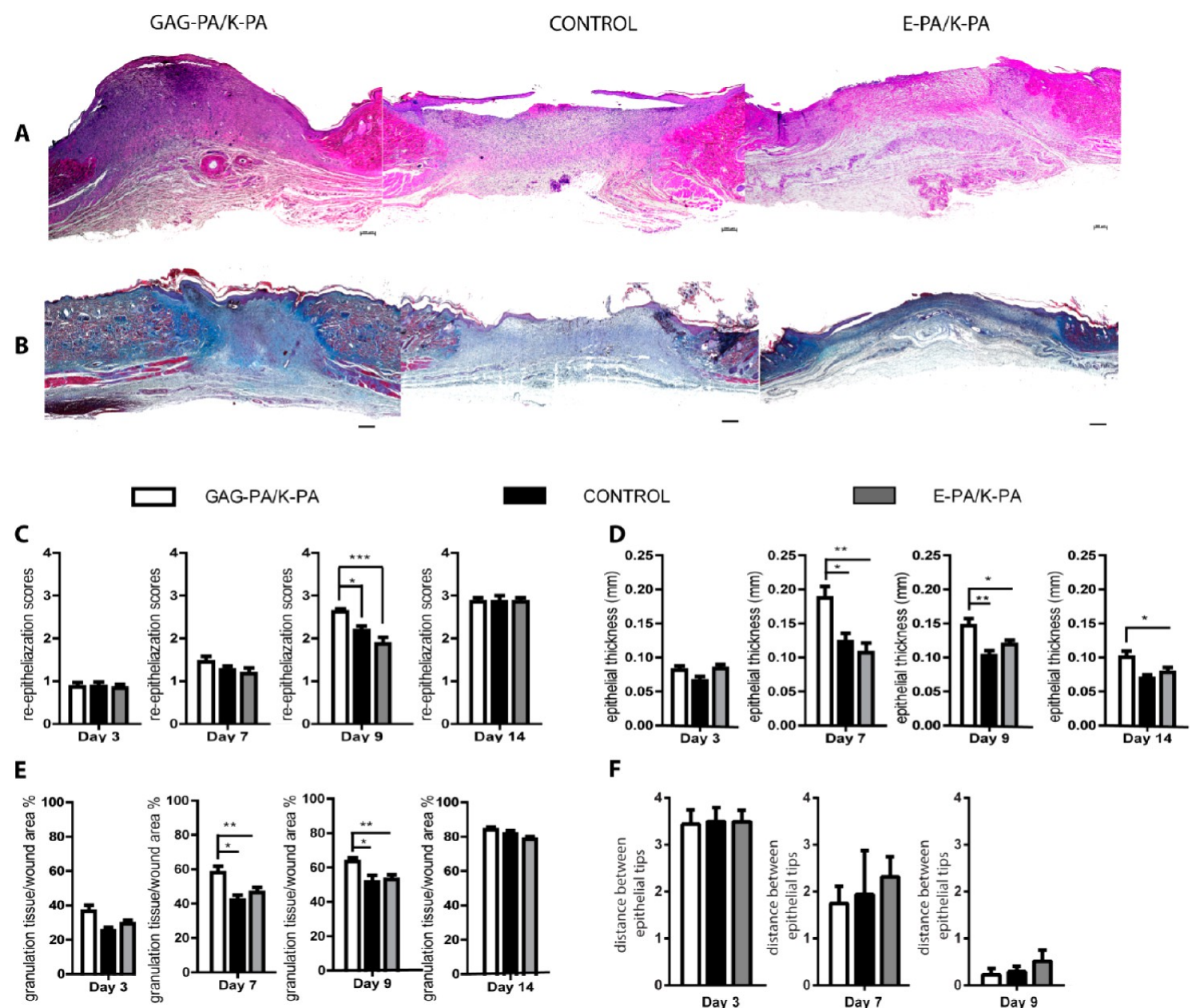

$\mathbf{F}$

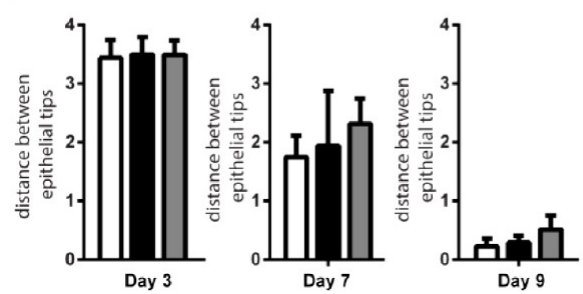

Figure 3. Bioactive gel application increased re-epithelialization, granulation tissue formation and collagen deposition in diabetic wounds. Light microscopy images of (A) H\&E and (B) Masson's trichrome stained tissue sections at day 9. (C) Re-epithelialization scoring and (D) epithelial thicknesses of tissue sections. Quantitative analysis of (E) granulation tissue formation per total wound area and (F) distance between endothelial tips $(\mu \mathrm{m})$. One-way ANOVA analysis was performed. $* p<0.05, * * p<0.01$, and $* * * p<0.001$, scale bars $=200 \mu \mathrm{m}$.

3.2. Bioactive Gel Treatment Accelerates Wound Healing in Diabetic Rats. STZ-injected (45 mg/kg) rats consistently became hyperglycemic after 3 weeks of diabetic induction, and animals with blood glucose levels above 20 $\mathrm{mmol} / \mathrm{L}$ were used in wound healing experiments. The body masses and blood glucose levels of the animals showed no statistically significant difference until the end of the experimental periods $(p>0.05)$ (Figure S4).

Wound closure rates (\%) were calculated for each group to investigate whether GAG-PA/K-PA gel treatment accelerates wound closure in diabetic rats. Following excision of fullthickness skin lesions, no significant difference was observed in wound closure between the three groups by day 3 . At days 7, 9 and 14, wound closure was significantly accelerated in GAG$\mathrm{PA} / \mathrm{K}-\mathrm{PA}$ treated groups compared to E-PA/K-PA and PBS treated controls (Figure 2A-E). Bioactive gel treated rats exhibited rapid wound closure after day 3 and approached nearly $80 \%$ of closure by day 14 postinjury. In contrast, E-PA/ $\mathrm{K}-\mathrm{PA}$ and PBS treated diabetic control rats exhibited impaired closure.

3.3. Bioactive Gel Treatment Accelerates Re-epithelialization, Granulation Tissue Formation, and Collagen Deposition. Keratinocyte proliferation and migration are critical for optimal wound healing, not only because of their role in the proliferation phase but also for their importance in the remodeling of skin tissue. Re-epithelialization scoring, epithelial thickness measurement and granulation tissue area calculation were performed to analyze the healing rate of wounds in diabetic rats (Figure 3). Although progressions of reepithelialization were similar between the groups on day 7 , GAG-PA/K-PA treated wounds exhibited nearly complete reepithelialization compared to moderate epithelialization of controls on day 9 (Figure 3C). In E-PA/K-PA treated rats, the dermal closure rate was similar to that in PBS treated rats. In the later stages of wound healing, however, re-epithelialization was complete for all groups (day 14). The distance between epithelial tips was calculated from each group (Figure 3F). Although not statistically significant, distance between epithelial tips was reduced in GAG-PA/K-PA treated groups at day 7 and 9. The epidermal thickness of regenerated skin was also measured for all groups to further investigate the effect of bioactive gel treatment on re-epithelialization. The thickness of bioactive PA treated epidermis was significantly greater than control groups on days 7 and 9 (Figure 3D). Dermal regeneration was further characterized by visualization of granulation tissue formation both with $\mathrm{H} \& \mathrm{E}$ and Massons's trichrome staining on day 9 (Figure 3A, B). Light microscopic examination on day 7 showed that granulation tissue per total wound area was $58 \%$ in the GAG-PA/K-PA treated groups whereas it was around $45 \%$ for other groups. At days 7 and 9 , 

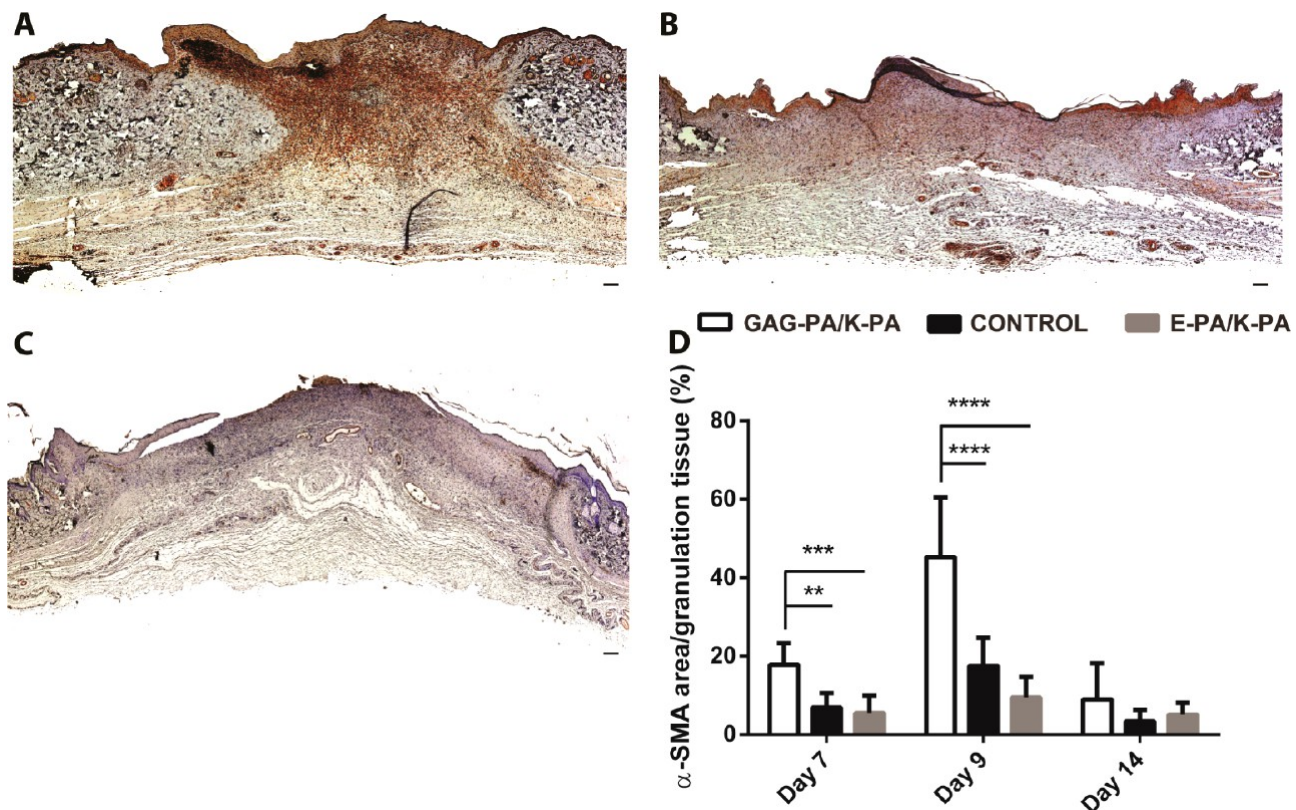

Figure 4. GAG-PA/K-PA treatment induced alpha smooth muscle actin expression in diabetic wounds. $\alpha$-SMA staining (brown) of wound tissues on day 9 treated with (A) GAG-PA/K-PA, (B) control, and (C) E-PA/K-PA, with hematoxylin counterstaining of nuclei (blue). (D) Quantification of percentage of $\alpha$-SMA positive staining per granulation area at indicated time points (day 7, 9, and 14) post wounding. Two-way ANOVA was performed. $* p<0.05$, ** $p<0.01$, and $* * * p<0.001$, scale bars $=200 \mu \mathrm{m}$.
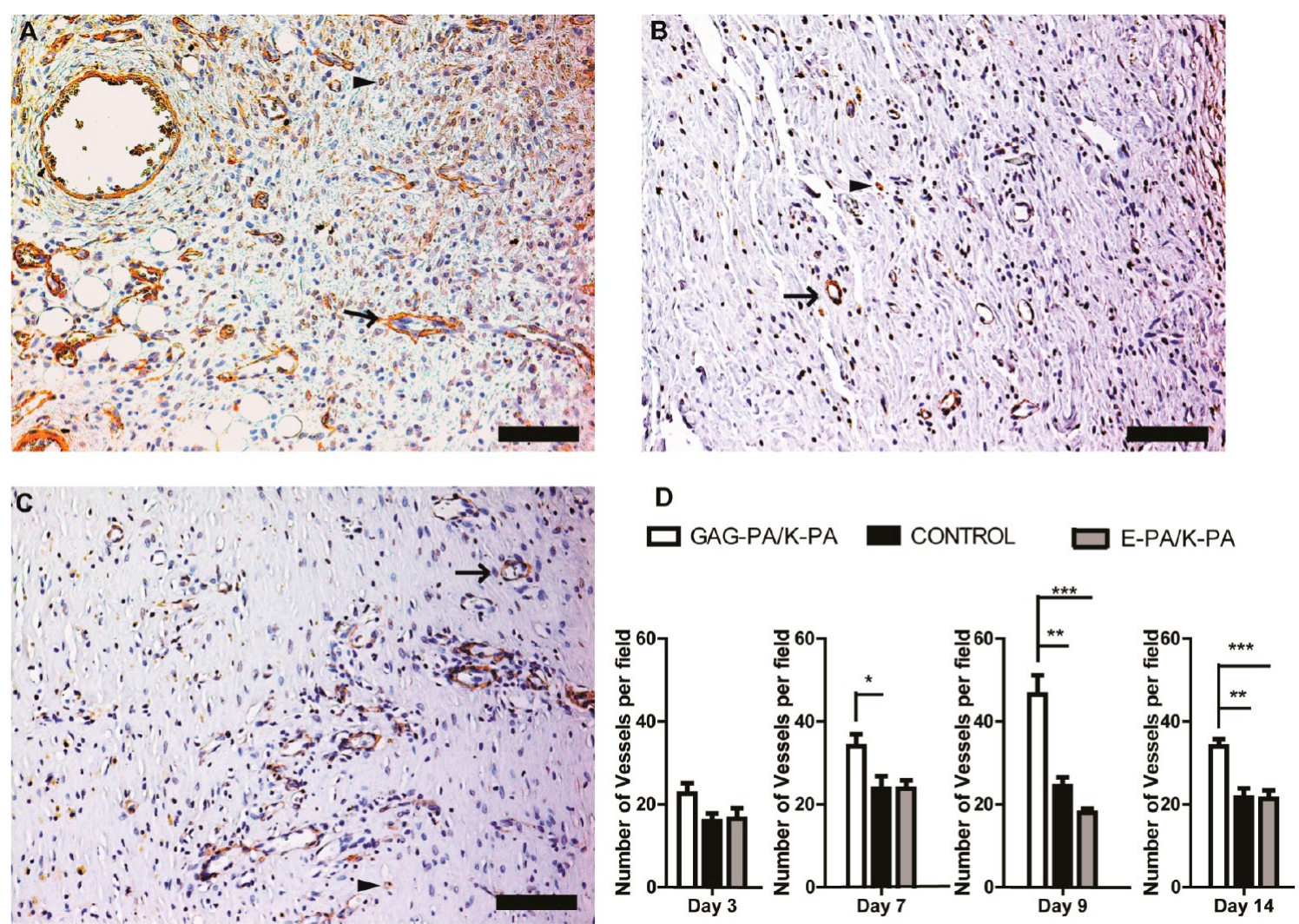

Figure 5. Accelerated blood vessel intensity in GAG-PA/K-PA treated wound areas suggests that heparin mimetic peptide nanofiber treatment induced angiogenesis. Representative von Willebrand factor staining (brown) images of wounds treated with (A) GAG-PA/K-PA, (B) PBS control, and (C) E-PA/K-PA at day 9 and hematoxylin-blue counterstaining of nuclei. Representative examples of large vessels (arrow) as well as capillaries (arrowheads) are shown in A-C. Quantitative analysis of number of vessels per field (D) was performed from images. Data are presented as means \pm SEM $* p<0.05, * * p<0.01, * * * p<0.001$, scale bars $=100 \mu \mathrm{m}$.

granulation tissue area per total wound area was significantly higher in GAG-PA/K-PA treated group (Figure 3E).
Furthermore, Massons's trichrome staining suggests that control and E-PA/K-PA treated sections have few and poorly 

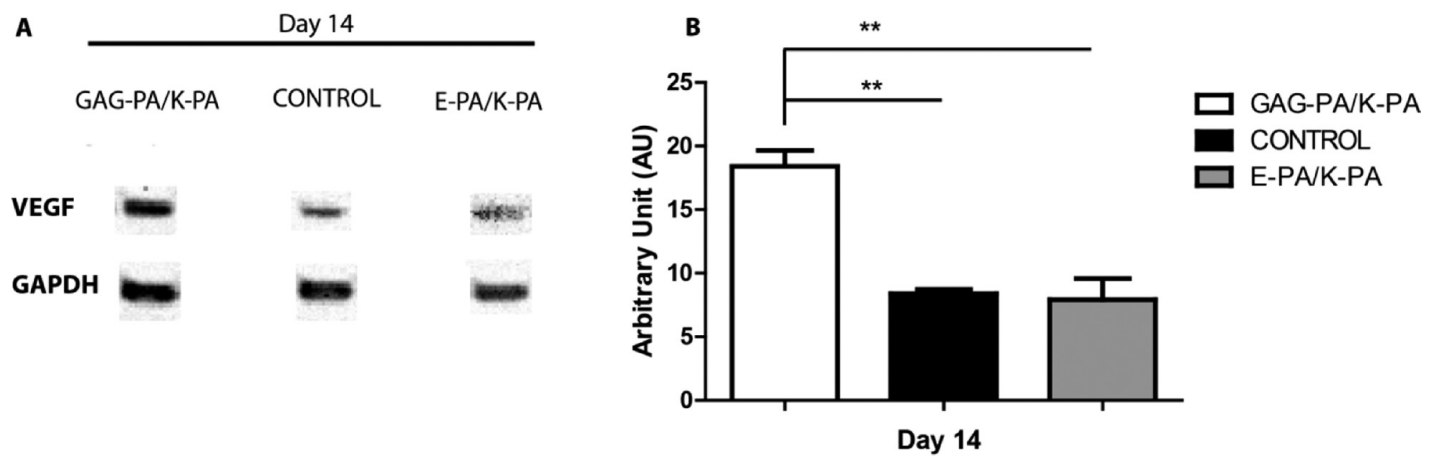

Figure 6. VEGF expression was enhanced in GAG-PA/K-PA-treated animals, whereas lower expression was observed in control and nonbioactive PA treated samples on day 14. The density of the bands was evaluated by ImageJ and normalized to GAPDH signal. Western blot analysis showed that VEGF expression in the GAG-PA/K-PA-treated group was almost two folds higher than controls. Data are presented as means \pm SEM $* * p<$ 0.01 .
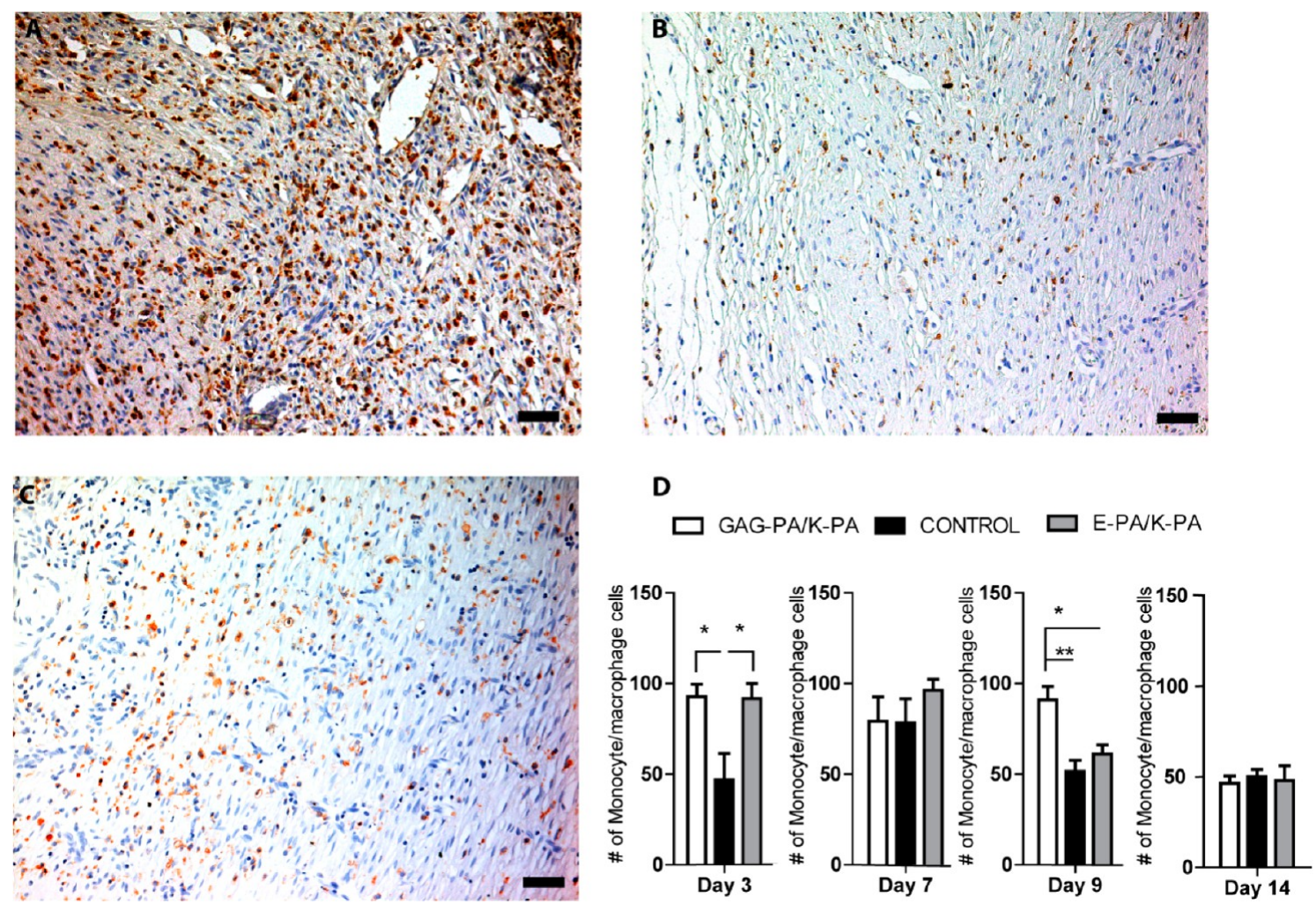

Figure 7. Monocyte/macrophage infiltration was increased in GAG-PA/K-PA treated wounds on day 9. CD68 immunostaining for (A) bioactive gel, (B) PBS control, and (C) nonbioactive gel treated group. Brown color indicates presence of monocyte/macrophage and hematoxylin-blue counterstaining of nuclei. (D) Quantitative analysis of number of monocyte/macrophage cells on days 3, 7, 9, and 14 post wounding. Data are presented as means \pm SEM $* p<0.05, * * p<0.01$, scale bars $=50 \mu \mathrm{m}$.

arranged collagen bundles at the wound site on day 9 (Figure $3 B$ ). On the other hand, granulation tissue of GAG-PA/K-PA treated wounds contained thicker and more-organized collagen fibrils and bundles compared to controls (Figure S5). Although there was a dramatic difference of collagen organization between bioactive gel and controls on day 9, collagen deposition occurs in all groups on day 14 (Figure S6).

3.4. GAG-PA/K-PA Treatment Induces Myofibroblast Activation. Tissue sections were stained with alpha smooth muscle actin (SMA) to monitor the transformation of fibroblast cells to into myofibroblasts in the wound area. At day 9, immunostaining of alpha SMA, showed that activated fibroblasts were abundantly located at the wound margins (Figure 4A-C). The positive immunostaining of alpha smooth muscle actin per granulation tissue was quantified at days 7 and 9 (Figure 4D). The results of this analysis indicated that the number of myofibroblasts and intensity of staining were significantly higher in the granulation tissue area of GAG-PA/ K-PA-treated wounds compared to controls.

3.5. Heparin Mimetic PA Nanofibers Induce Robust Neovascularization in Diabetic Wounds. Since angiogenesis is a crucial event in wound healing especially for the closure of chronic wounds, we examined the efficacy of heparin mimetic peptide nanofibers to induce the neovascularization in diabetic skin tissue (Figure 5A-C). As evaluated by serial sectioning, total number of vessels per field markedly increased in heparin mimetic peptide nanofiber treated rats at days 7, 9, and 14 (Figure 5D). In addition to the quantification of vessel 
numbers, histological analysis showed that large vacuolar-like vessels were seen in the wound bed of GAG-PA/K-PA-treated tissue sections (Figure 5A). Next, we tested whether heparin mimetic peptide nanofibers increase VEGF secretion to identify the mechanisms underlying the effects of these gels on promoting number of blood vessel formation.

3.6. Heparin Mimetic PA Nanofibers Promote Angiogenesis via Induction of VEGF Expression. VEGF protein expression was quantified in diabetic skin wounds by Western blot analysis at day 7 (early response) and day 14 (late response) to understand the basis of the enhanced angiogenesis in on GAG-PA/K-PA treated animals. No significant difference was found at VEGF protein level between groups on day 7 by Western blot analysis (Figure S7). Higher expressions of VEGF were observed in GAG-PA/K-PA treated animals on day 14 compared to both E-PA/K-PA and PBS control animals (Figure $6 \mathrm{~A}, \mathrm{~B})$. As such, it is likely that VEGF expression was stimulated at later days of recovery by the GAG-PA/K-PA system.

3.7. Bioactive Gel Treatment Affects Infiltration of Inflammatory Cells. Characterization of inflammatory stage of wounds was performed by CD68 (monocyte/macrophage marker) staining (Figure 7A-C). Quantitative analysis showed that number of macrophages was higher in both heparin mimetic GAG-PA/K-PA $(92.6 \pm 6.8)$ and nonbioactive E-PA/ K-PA $(91.6 \pm 8.2)$ peptide nanofiber treated groups in contrast to PBS treated control $(46.7 \pm 14.5)$ animals on day 3 (Figure $7 D)$. Although the number of macrophage was 2 -fold higher than PBS control on early onset, no difference was found on day 7. Moreover, a gradual increase in monocyte/macrophage number was observed in GAG-PA/K-PA group on day 9 .

\section{DISCUSSION}

Vascular complications that eventually lead to unhealed wound ulcers are characteristic of diabetes. Reduced expression of growth factors and impairment of angiogenesis are the main problems associated with diabetic foot ulcers. In this study, heparin mimetic peptide nanofibers were found to enhance wound healing rate by accelerating angiogenesis, promoting reepithelialization process and stimulating the expression of VEGF. This finding correlates with studies that report that heparin-binding structures exhibit positive effects on wound healing. ${ }^{19,20}$

Animal wound healing models are important biological tools for understanding the cellular and molecular bases of tissue repair and play a vital role in the development and validation of potential strategies for clinical treatment. Their optimization is therefore important for the determination of the effectiveness of a prospective material or treatment method. While transgenic animals are ideal for the investigation of the diabetic wound healing process, there is an increasing amount of evidence that shows that STZ administration can create a suitable model of type I diabetes for wound healing studies. $^{21-23}$ In the present study, we have also implemented minor modifications to our experimental protocol to better accommodate the properties of the peptide amphiphile gels used. In particular, a preliminary study had shown that gels in uncovered wound areas may leak from the wound and mix with each other because of the movement of the animals; as such, potential gel mixing was prevented by coating the wounds with a nonwoven, hypoallergenic, air-permeable elastic tape.

The main function of heparan sulfate proteoglycans (HSPGs) is their ability to modulate angiogenesis by binding to VEGF. ${ }^{24}$ VEGF has an extracellular matrix (ECM)-binding domain and possesses binding affinity for HSPGs. ${ }^{25}$ In our previous studies, we presented a heparin mimetic peptide amphiphile (PA) nanofiber system that mimics the function of heparan sulfate by presenting optimal arrangement of functional groups $\left(-\mathrm{COOH},-\mathrm{OH}\right.$ and $\left.-\mathrm{SO}_{3}\right)$ on nanofibrous structures. ${ }^{16}$ In these previous studies, we also showed that the presence of all of these functional groups within the same system is critical for growth factor binding, and that control peptides containing only one or two of these groups exhibit limited functionality compared to GAG-PA. ${ }^{16}$ Furthermore, high binding affinity of peptide system to $\mathrm{VEGF}_{165}$ rather than VEGF $_{121}$ (which lacks heparin-binding domain) showed that growth factor binding was site specific. ${ }^{26}$ These studies also demonstrated that this nanofiber system showed better growth factor binding, enhanced induction of angiogenesis and neurogenesis than control PA nanofibers in vitro. ${ }^{16,26,27}$ Our present findings showed that wound healing rate was accelerated in the GAG-PA/K-PA treated group compared to PBS control and nonbioactive peptide treated groups due to enhanced growth factor activity. As such, the increase in regenerative capacity is attributable to the amino acid sequence of the heparin mimetic group rather than the nanofibrous structures of the peptide amphiphile systems.

In diabetes, phases of wound healing process do not occur in synchrony, due to complications of the disease. We analyzed the progress in wound healing at different time points (days 3, 7,9 , and 14) along 2 weeks after treatment application. We found that gel treatment does not cause any difference on healing rate at the first stage of wound healing process (hemostasis), as the diabetic wounds began to release cytokines and growth factors for cell recruitment. ${ }^{4}$ On the other hand, heparin mimetic hydrogel treatment enhanced wound healing at the inflammation and the proliferation phases.

CD68 staining was performed to observe monocyte/ macrophage recruitment to the wound area and to investigate the extent of the immune response during the inflammation phase. The presence of macrophage was required not only for efficient wound repair but also for induction of neovascularization. ${ }^{28,29}$ Reduced and abnormal vascularization was found in wound of macrophage deficient mice. ${ }^{30,31}$ The nanofibrous structure of the scaffolds might be one factor that induces the early infiltration of monocyte/macrophage cells to the wound area, as both bioactive and nonbioactive PA-treated wounds exhibited heightened inflammatory responses on day 3; however, this scaffold effect was temporary (Figure 7D). On day 7, PBS treated rats had also similar number of monocyte/ macrophage with gel treated rats. Inflammatory response was significantly higher in GAG-PA/K-PA treated group on day 9 correlated with enhanced neovascularization (Figure 5D). In contrast, nonbioactive gel and PBS-treated rats exhibited diminished expression of macrophages on day 9. In an efficient wound healing process, wound macrophages disappear at the end of inflammatory phase. ${ }^{28}$ The reduction in the higher number of monocyte/macrophages in the GAG-PA/K-PAtreated group on day 14 indicated that macrophage infiltration ceased and left the wound area.

Keratinocyte migration and differentiation are inhibited in diabetic wounds. ${ }^{32}$ Interaction of keratinocytes with fibroblasts is crucial for proper wound healing as these interactions induce the secretion of growth factors and cytokines. ${ }^{33}$ The results presented here show that heparin mimetic hydrogel treatment recruits keratinocytes to leading edge and allows their persistent 
migration. The proliferation phase is also compromised in diabetic wounds; ${ }^{34}$ however, re-epithelialization scoring and epithelial thicknesses results in this study indicate that tissue proliferation was promoted in heparin mimetic hydrogel treated wounds.

Myofibroblasts are specialized fibroblasts that play important roles in collagen synthesis and scar formation. ${ }^{35}$ During wound healing, myofibroblasts develop from granulation tissue and transiently express $\alpha$-SMA. ${ }^{36}$ Bioactive gel treatment was found to recruit cells to the myofibroblastic phenotype, which is essential for remodeling during the wound healing process. It is notable that $\alpha$-SMA expression was significantly higher in the heparin mimetic group on day 7 and 9 (Figure 4). This result is also in correlation with the observed increase in granulation tissue formation (Figure 3A-F). VEGF expression is known to induce myofibroblast transformation through the activation of TGF- $\beta 1{ }^{37}$ We have also observed a 2 -fold increase in VEGF expression in heparin mimetic PA-treated animals compared to control (Figure 6). Thus, the significant myofibroblast presence and positive SMA staining in GAG-PA/K-PA-treated wounds may be due to stronger VEGF expression in the wound area.

VEGF is essential for early blood vessel formation and angiogenesis. ${ }^{38}$ Wounds of STZ induced rats had diminished expression of VEGF compared to nondiabetic wounds. ${ }^{39}$ Western blot analysis showed that VEGF expression was similar between samples on day 7 (Figure S8), but increased in GAG-PA/K-PA treated wounds on day 14 (Figure 6). During the wound healing process, VEGF expression was obtained from many cell types such as endothelial cells, fibroblasts, smooth muscle cells, platelets, neutrophils, and macrophages. ${ }^{5}$ The presence of high number of those cells in the wound area contributes VEGF secretion upon heparin mimetic gel treatment.

Increases in the number of newly formed vessels, together with high VEGF expression patterns, suggest that heparin mimetic gels are able to protect and stabilize the wound environment through modulation of angiogenesis. We also demonstrated that nonbioactive fibers had little to no influence on wound healing, except for an increase in early macrophage recruitment a nonsignificant trend toward delayed recovery, suggesting that the effects observed in the GAG-PA/K-PA group were sequence-specific. Overall, heparin mimetic bioactive peptide nanofiber gels were found to be effective in increasing VEGF expression and promoting vessel formation in diabetic wounds.

\section{CONCLUSION}

In this study, we show the application of a previously developed heparin mimetic peptide nanofiber system for the treatment of diabetic wounds. This bioinspired material promoted wound healing in the STZ-induced diabetic rat wound model. Results presented in this study showed that heparin mimetic peptide nanofiber treatment has a significant impact on the inflammation and proliferation phases of the healing process. The application of the bioactive gel formed by these peptide nanofibers to wound area promotes angiogenesis, re-epithelialization, and inflammatory response. In addition, cell recruitment was accelerated and macrophage infiltration was increased during the inflammatory phase in GAG-PA/K-PA treated rats. A marked increase on expression of VEGF and number of blood vessels are strong evidence of the induction of angiogenesis in diabetic wounds. Overall, these results show that heparin mimetic peptide nanofibers are a promising therapeutic platform for the treatment of dermal wounds in diabetes patients.

\section{ASSOCIATED CONTENT}

\section{Supporting Information}

The Supporting Information is available free of charge on the ACS Publications website at DOI: 10.1021/acsbiomaterials.6b00238.

Supporting figures, liquid chromatography, and mass spectroscopy; SEM images; circular dichroism (CD); changes in body weights and blood glucose level; Masson's Trichrome staining; the distance between epithelial tips; Western blot analysis on day 7 (PDF)

\section{AUTHOR INFORMATION}

\section{Corresponding Authors}

*E-mail: delibasi@hacettepe.edu.tr.

*E-mail: moguler@unam.bilkent.edu.tr.

*E-mail: atekinay@bilkent.edu.tr.

\section{Notes}

The authors declare no competing financial interest.

\section{ACKNOWLEDGMENTS}

This work was supported by the Scientific and Technological Research Council of Turkey (TUBITAK) grant number 114S912. B.S. is supported by TUBITAK BIDEB PhD fellowship. The authors thank Ibrahim Ulusoy for his gracious help in postoperatory care of animals, Alper Devrim Ozkan for critical reading, and Elif Arslan for SEM images.

\section{REFERENCES}

(1) Association, A. D. Diagnosis and classification of diabetes mellitus. Diabetes Care 2009, 32 (Suppl 1), S62-7.

(2) Adeghate, E.; Schattner, P.; Dunn, E. An update on the etiology and epidemiology of diabetes mellitus. Ann. N. Y. Acad. Sci. 2006, 1084, $1-29$.

(3) Brem, H.; Tomic-Canic, M. Cellular and molecular basis of wound healing in diabetes. J. Clin. Invest. 2007, 117 (5), 1219-22.

(4) Falanga, V. Wound healing and its impairment in the diabetic foot. Lancet 2005, 366 (9498), 1736-43.

(5) Bao, P.; Kodra, A.; Tomic-Canic, M.; Golinko, M. S.; Ehrlich, H. P.; Brem, $\mathrm{H}$. The role of vascular endothelial growth factor in wound healing. J. Surg. Res. 2009, 153 (2), 347-58.

(6) Tandara, A. A.; Mustoe, T. A. Oxygen in wound healing-more than a nutrient. World J. Surg. 2004, 28 (3), 294-300.

(7) Tonnesen, M. G.; Feng, X.; Clark, R. A. Angiogenesis in wound healing. J. Invest. Dermatol. Symp. Proc. 2000, 5 (1), 40-6.

(8) Thangarajah, H.; Yao, D.; Chang, E. I.; Shi, Y.; Jazayeri, L.; Vial, I. N.; Galiano, R. D.; Du, X. L.; Grogan, R.; Galvez, M. G.; Januszyk, M.; Brownlee, M.; Gurtner, G. C. The molecular basis for impaired hypoxia-induced VEGF expression in diabetic tissues. Proc. Natl. Acad. Sci. U. S. A. 2009, 106 (32), 13505-10.

(9) Eming, S. A.; Krieg, T. Molecular mechanisms of VEGF-A action during tissue repair. J. Invest. Dermatol. Symp. Proc. 2006, 11 (1), 7986.

(10) Galiano, R. D.; Tepper, O. M.; Pelo, C. R.; Bhatt, K. A.; Callaghan, M.; Bastidas, N.; Bunting, S.; Steinmetz, H. G.; Gurtner, G. C. Topical vascular endothelial growth factor accelerates diabetic wound healing through increased angiogenesis and by mobilizing and recruiting bone marrow-derived cells. Am. J. Pathol. 2004, 164 (6), 1935-47.

(11) Christianson, H. C.; Belting, M. Heparan sulfate proteoglycan as a cell-surface endocytosis receptor. Matrix Biol. 2014, 35, 51-5. 
(12) Zhao, W.; McCallum, S. A.; Xiao, Z.; Zhang, F.; Linhardt, R. J. Binding affinities of vascular endothelial growth factor (VEGF) for heparin-derived oligosaccharides. Biosci. Rep. 2012, 32 (1), 71-81.

(13) Moura, L. I.; Dias, A. M.; Carvalho, E.; de Sousa, H. C. Recent advances on the development of wound dressings for diabetic foot ulcer treatment-a review. Acta Biomater. 2013, 9 (7), 7093-114.

(14) Demidova-Rice, T. N.; Hamblin, M. R; Herman, I. M. Acute and impaired wound healing: pathophysiology and current methods for drug delivery, part 2: role of growth factors in normal and pathological wound healing: therapeutic potential and methods of delivery. Adv. Skin Wound Care 2012, 25 (8), 349-70.

(15) Cui, H.; Webber, M. J.; Stupp, S. I. Self-assembly of peptide amphiphiles: from molecules to nanostructures to biomaterials. Biopolymers 2010, 94 (1), 1-18.

(16) Mammadov, R.; Mammadov, B.; Toksoz, S.; Aydin, B.; Yagci, R.; Tekinay, A. B.; Guler, M. O. Heparin mimetic peptide nanofibers promote angiogenesis. Biomacromolecules 2011, 12 (10), 3508-19.

(17) Tibbitt, M. W.; Anseth, K. S. Hydrogels as extracellular matrix mimics for 3D cell culture. Biotechnol. Bioeng. 2009, 103 (4), 655-63.

(18) Greenfield, N. J. Using circular dichroism spectra to estimate protein secondary structure. Nat. Protoc. 2007, 1 (6), 2876-2890.

(19) Tong, M.; Tuk, B.; Shang, P.; Hekking, I. M.; Fijneman, E. M.; Guijt, M.; Hovius, S. E.; van Neck, J. W. Diabetes-impaired wound healing is improved by matrix therapy with heparan sulfate glycosaminoglycan mimetic OTR4120 in rats. Diabetes 2012, 61 (10), 2633-41.

(20) Rajangam, K.; Behanna, H. A.; Hui, M. J.; Han, X.; Hulvat, J. F.; Lomasney, J. W.; Stupp, S. I. Heparin binding nanostructures to promote growth of blood vessels. Nano Lett. 2006, 6 (9), 2086-90.

(21) Moura, L. I.; Dias, A. M.; Leal, E. C.; Carvalho, L.; de Sousa, H. C.; Carvalho, E. Chitosan-based dressings loaded with neurotensin-an efficient strategy to improve early diabetic wound healing. Acta Biomater. 2014, 10 (2), 843-57.

(22) Kim, H. S.; Yoo, H. S. In vitro and in vivo epidermal growth factor gene therapy for diabetic ulcers with electrospun fibrous meshes. Acta Biomater. 2013, 9 (7), 7371-80.

(23) Bloch, K.; Vanichkin, A.; Damshkaln, L. G.; Lozinsky, V. I.; Vardi, P. Vascularization of wide pore agarose-gelatin cryogel scaffolds implanted subcutaneously in diabetic and non-diabetic mice. Acta Biomater. 2010, 6 (3), 1200-5.

(24) Chiodelli, P.; Mitola, S.; Ravelli, C.; Oreste, P.; Rusnati, M.; Presta, M. Heparan sulfate proteoglycans mediate the angiogenic activity of the vascular endothelial growth factor receptor-2 agonist gremlin. Arterioscler., Thromb., Vasc. Biol. 2011, 31 (12), e116-27.

(25) Miralem, T.; Steinberg, R.; Price, D.; Avraham, H. VEGF(165) requires extracellular matrix components to induce mitogenic effects and migratory response in breast cancer cells. Oncogene 2001, 20 (39), 5511-24.

(26) Mammadov, R.; Mammadov, B.; Guler, M. O.; Tekinay, A. B. Growth factor binding on heparin mimetic peptide nanofibers. Biomacromolecules 2012, 13 (10), 3311-9.

(27) Mammadov, B.; Mammadov, R; Guler, M. O.; Tekinay, A. B. Cooperative effect of heparan sulfate and laminin mimetic peptide nanofibers on the promotion of neurite outgrowth. Acta Biomater. 2012, 8 (6), 2077-86.

(28) Brancato, S. K.; Albina, J. E. Wound macrophages as key regulators of repair: origin, phenotype, and function. Am. J. Pathol. 2011, 178 (1), 19-25.

(29) Eming, S. A.; Krieg, T.; Davidson, J. M. Inflammation in wound repair: molecular and cellular mechanisms. J. Invest. Dermatol. 2007, 127 (3), 514-25.

(30) Mirza, R.; DiPietro, L. A.; Koh, T. J. Selective and specific macrophage ablation is detrimental to wound healing in mice. Am. J. Pathol. 2009, 175 (6), 2454-62.

(31) Goren, I.; Allmann, N.; Yogev, N.; Schürmann, C.; Linke, A.; Holdener, M.; Waisman, A.; Pfeilschifter, J.; Frank, S. A transgenic mouse model of inducible macrophage depletion: effects of diphtheria toxin-driven lysozyme $\mathrm{M}$-specific cell lineage ablation on wound inflammatory, angiogenic, and contractive processes. Am. J. Pathol. 2009, 175 (1), 132-47.

(32) Usui, M. L.; Mansbridge, J. N.; Carter, W. G.; Fujita, M.; Olerud, J. E. Keratinocyte migration, proliferation, and differentiation in chronic ulcers from patients with diabetes and normal wounds. $J$. Histochem. Cytochem. 2008, 56 (7), 687-96.

(33) Menon, S. N.; Flegg, J. A.; McCue, S. W.; Schugart, R. C.; Dawson, R. A.; McElwain, D. L. Modelling the interaction of keratinocytes and fibroblasts during normal and abnormal wound healing processes. Proc. R. Soc. London, Ser. B 2012, 279 (1741), 3329-38.

(34) Xu, F.; Zhang, C.; Graves, D. T. Abnormal cell responses and role of TNF- $\alpha$ in impaired diabetic wound healing. BioMed Res. Int. 2013, 2013, 754802.

(35) Hinz, B. Formation and function of the myofibroblast during tissue repair. J. Invest. Dermatol. 2007, 127 (3), 526-37.

(36) Darby, I.; Skalli, O.; Gabbiani, G. Alpha-smooth muscle actin is transiently expressed by myofibroblasts during experimental wound healing. Lab Invest. 1990, 63 (1), 21-29.

(37) Park, H. Y.; Kim, J. H.; Park, C. K. VEGF induces TGF- $\beta 1$ expression and myofibroblast transformation after glaucoma surgery. Am. J. Pathol. 2013, 182 (6), 2147-54.

(38) Hoeben, A.; Landuyt, B.; Highley, M. S.; Wildiers, H.; Van Oosterom, A. T.; De Bruijn, E. A. Vascular endothelial growth factor and angiogenesis. Pharmacol. Rev. 2004, 56 (4), 549-580.

(39) Shukla, A.; Dubey, M. P.; Srivastava, R.; Srivastava, B. S. Differential expression of proteins during healing of cutaneous wounds in experimental normal and chronic models. Biochem. Biophys. Res. Commun. 1998, 244 (2), 434-9. 